\title{
Separation of piezoelectric grain resonance and domain wall dispersion in $\mathrm{Pb}(\mathrm{Zr}, \mathrm{Ti}) \mathrm{O}_{3}$ ceramics
}

\author{
V. Porokhonskyy, ${ }^{\text {a) }}$ Li Jin (靳立), and D. Damjanovic \\ Ceramics Laboratory, Swiss Federal Institute of Technology (EPFL), CH-1015 Lausanne, Switzerland
}

(Received 27 March 2009; accepted 6 May 2009; published online 29 May 2009)

\begin{abstract}
We report on the experimental investigation of a high-frequency $(1 \mathrm{MHz}-1.8 \mathrm{GHz})$ dielectric dispersion in unpoled and poled $\mathrm{Pb}(\mathrm{Zr}, \mathrm{Ti}) \mathrm{O}_{3}$ ceramics. Two overlapping loss peaks could be revealed in the dielectric spectrum. The linear dependence between the lower-frequency peak position and average grain size $\bar{D}$, which holds for $\bar{D} \leq 10 \mu \mathrm{m}$, indicates that the corresponding polarization mechanism originates from piezoelectric resonances of grains. The intensity of the higher-frequency peak is drastically reduced by poling. It is thus proposed that this loss peak is related to domain wall contribution to the dielectric dispersion. (C) 2009 American Institute of Physics. [DOI: 10.1063/1.3147166]
\end{abstract}

It has been often observed that the lattice permittivity of ferroelectric materials is significantly lower than the permittivity measured at conventionally accessible frequencies, say below $1 \mathrm{MHz}$. Perhaps the most striking manifestation of this difference is seen in the strong, steplike dispersion of the permittivity accompanied by the loss peak in the frequency range from $10^{8}$ to $10^{10} \mathrm{~Hz}^{1-3}$ Above this frequency range, the permittivity is close to that of the lattice. Several mechanisms have been proposed to explain origins of the dispersion, including resonance of domain walls, ${ }^{4}$ translational vibration of domain walls, ${ }^{5}$ acoustic shear waves generation by stacks of lamellar $90^{\circ}$ domains, ${ }^{6}$ and piezoelectric resonance of grains. ${ }^{7}$ Some authors have proposed that the dispersion is related to motion of individual ions between off-center position. ${ }^{8}$ Being quite far from the state that permits quantitative description, the proposed mechanisms are sometimes arbitrarily invoked to explain any kind of dispersion observed in this frequency range, thus causing controversy and confusion. Most studies usually assign only one mechanism to the high frequency dispersion in a given ferroelectric material. ${ }^{4,6-8}$ However, there is no reason why several dispersive processes cannot contribute to the permittivity simultaneously. This is particularly true for piezoelectric resonances of grains and mechanisms related to domain wall displacement. Domain size (distance between neighboring domain walls) and grain size are coupled ${ }^{9}$ and their contribution to the dielectric dispersion may occur in the narrow frequency range. The model of Pertsev and Arlt, ${ }^{5}$ for example, suggests that resonance frequency of domain walls may be lower than the frequency of the elastic resonance of grains. The aim of this investigation is thus to specifically address the contribution of grain resonances and domain walls to the dielectric dispersion in $10^{8}$ to $10^{10} \mathrm{~Hz}$ range in technologically important ferroelectric, $\mathrm{Pb}(\mathrm{Zr}, \mathrm{Ti}) \mathrm{O}_{3}$ or PZT.

In order to obtain samples with broad range of average grain size, we take advantage of the fact that in PZT grain size can be significantly affected by dopants. ${ }^{10}$ Therefore, the samples of hard and soft PZT ceramics were prepared by a conventional solid state process using standard mixed oxide route. The $\mathrm{Zr} / \mathrm{Ti}$ ratio (in at. \%) was set to 58/42, which

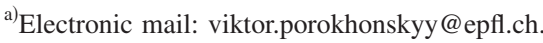

corresponds to a pure rhombohedral phase at room temperature. Hardening and softening effects were achieved by partial $B$-site substitution by $\mathrm{Fe}^{3+}$ or $\mathrm{Nb}^{5+}$, respectively. Further details on the sample synthesis can be found elsewhere. ${ }^{10,11}$ For the sake of convenience, the samples are labeled in the following manner: for example, PZT(58/42)Fe1.0 refers to $\mathrm{Pb}\left(\mathrm{Zr}_{0.58} \mathrm{Ti}_{0.42}\right)_{0.99} \mathrm{Fe}_{0.01} \mathrm{O}_{3}$, etc.

To determine grain size, the scanning electron microscopy images collected on polished and thermally etched samples were processed by means of IMAGEJ software package. ${ }^{12}$ As a result we obtained the cross-section area on the plane of polish $A_{c s}$ for individual grains. Further, the equivalent grain diameter was introduced as $D_{e}=2 \sqrt{A_{c s} / \pi}$. The distribution of $D_{e}$ was found to be unimodal for all compositions. Corresponding probability plots reveal quite good agreement with the log-normal distribution for a number of samples, while minor right-skew deviations were evident for some samples. At this point, one needs to note that $A_{c s}$ and $D_{e}$ describe random cross section of a grain. It is obvious, therefore, that they underestimate its threedimensional extent. To overcome this problem we adopt here the approach developed in Ref. 13. Assuming particular grain shape, it relates average grain size $\bar{D}$ to the average intercept length $\bar{L}$ by a proportionality constant. In the present study, 1.56 was used as such correction factor, ${ }^{13}$ which was applied to the average value of $D_{e}$, instead of $\bar{L}$. It is justified in our view, as $\bar{D}_{e}$ turns out to be very close to roughly estimated $\bar{L}$, as evident from Table I.

For dielectric characterization, poling, and electromechanical measurements, the samples were shaped into the form of small cylinders, with typical dimensions of about 0.9 $\mathrm{mm}$ in diameter and $5 \mathrm{~mm}$ in length. The base surfaces were electroded. The impedance spectra in the frequency range from $1 \mathrm{MHz}$ to $1.8 \mathrm{GHz}$ were collected by means of HP4396A network analyzer. Nonuniform fields distribution across the sample has been accounted for during permittivity calculation. ${ }^{14}$ For each composition the dielectric spectra were collected first starting with a well aged unpoled state and then immediately after each poling step.

In unpoled state all samples, soft and hard alike, exhibited strong dispersion in agreement with earlier studies. ${ }^{1,2}$ 
TABLE I. Microstructural and electromechanical properties of studied PZT samples.

\begin{tabular}{|c|c|c|c|c|c|c|c|c|c|c|}
\hline Composition & $\begin{array}{c}\bar{D}_{e} \\
(\mu \mathrm{m})\end{array}$ & $\begin{array}{c}\bar{L} \\
(\mu \mathrm{m})\end{array}$ & $\begin{array}{c}\bar{D} \\
(\mu \mathrm{m})\end{array}$ & $\begin{array}{c}f_{p} \\
(\mathrm{GHz})\end{array}$ & $\begin{array}{c}V_{s} \\
(\mathrm{~m} / \mathrm{s})\end{array}$ & $\begin{array}{c}d_{33} \\
(\mathrm{pC} / \mathrm{N})\end{array}$ & $k_{33}$ & $Q_{m}$ & $\begin{array}{c}\lambda_{A} \\
(\mu \mathrm{m})\end{array}$ & $\begin{array}{c}\text { Density } \\
(\%)\end{array}$ \\
\hline $\operatorname{PZT}(58 / 42) \mathrm{Fe} 1.0$ & 0.83 & 0.87 & 1.3 & 0.937 & 4073 & 77.8 & 0.51 & 59 & 4.35 & 97.6 \\
\hline $\operatorname{PZT}(58 / 42) \mathrm{Fe} 0.5$ & 2.13 & 2.02 & 3.32 & 0.485 & 4028 & 57.8 & 0.36 & 115 & 8.30 & 96.5 \\
\hline $\operatorname{PZT}(58 / 42) \mathrm{Nb} 1.0$ & 2.43 & 2.55 & 3.79 & 0.485 & 3987 & 102.7 & 0.6 & 57 & 8.22 & 95.3 \\
\hline $\operatorname{PZT}(58 / 42) \mathrm{Nb} 0.85$ & 2.63 & 2.58 & 4.1 & 0.398 & 3971 & 100 & 0.55 & 72 & 9.98 & 97.4 \\
\hline $\operatorname{PZT}(58 / 42) \mathrm{Nb} 0.7$ & 3.51 & 3.6 & 5.48 & 0.348 & 3971 & 93 & 0.53 & 73 & 11.41 & 97.7 \\
\hline $\operatorname{PZT}(58 / 42) \mathrm{Fe} 0.1$ & 3.89 & 3.98 & 6.21 & 0.306 & 3871 & 80.3 & 0.49 & 139 & 12.65 & 95.8 \\
\hline $\operatorname{PZT}(58 / 42)$ & 6.05 & 5.95 & 9.43 & 0.267 & 4103 & 90 & 0.61 & 50 & 15.37 & 99.7 \\
\hline $\operatorname{PZT}(58 / 42) \mathrm{Nb} 0.5$ & 6.74 & 6.71 & 10.51 & 0.180 & 3964 & 88 & 0.52 & 107 & 22.02 & 96.1 \\
\hline $\operatorname{PZT}(58 / 42) \mathrm{Nb} 0.2$ & 10.84 & 10.44 & 16.91 & 0.221 & 4415 & 77 & 0.67 & 66 & 19.98 & 96.1 \\
\hline
\end{tabular}

The loss spectra reveal single peak located from 0.7 to about 3.4 GHz. The upper bound of this range was determined by means of the sleeve resonator technique. ${ }^{15}$ The data are not shown here. Hereafter the frequency of corresponding loss maximum will be referred to as $f_{u}$. For poling the samples were exposed to the voltage equivalent of $25 \mathrm{kV} / \mathrm{cm}$ at $100-130{ }^{\circ} \mathrm{C}$. The field was kept on for at least $30 \mathrm{~min}$ at elevated temperature and during cooling. Once the samples had been poled, their electromechanical properties (e.g., coupling coefficient $k_{33}$, sound velocity $V_{s}$, etc.) were determined by means of piezoelectric resonance method ${ }^{16}$ employed in the longitudinal-bar mode. The results for $k_{33}$ (see Table I) agree with the values typically reported for PZT indicating that the samples were fully poled.

In well poled samples we observe single loss maximum. It is centered at somewhat lower frequencies (designated here $f_{p}$ ) compared to the peak position of corresponding unpoled state $f_{u}$. The separation between $f_{u}$ and $f_{p}$ amounts to at least $0.5 \mathrm{GHz}$. Figure 1 shows selected loss and permittivity spectra of poled samples. To learn more about the relationship between peaks in poled and unpoled ceramics, one of the samples, PZT(58/42) Nb0.5, was poled gradually. Its dielectric spectra collected first in unpoled state and then after each consecutive poling step are summarized in Fig. 2. Rapid permittivity and loss variations below $10 \mathrm{MHz}$, which are seen here in the last spectrum, are due to piezoelectric resonances of the sample. At higher frequencies each subse-

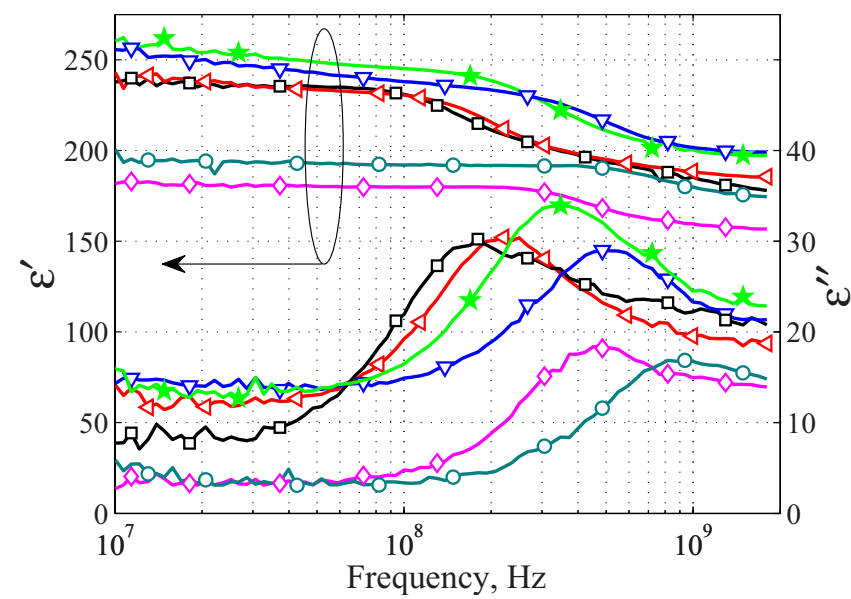

FIG. 1. (Color online) Permittivity and loss spectra of well poled samples. The symbols are introduced solely to facilitate distinction between different

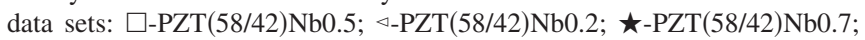
$\nabla$-PZT(58/42)Nb1.0; $\diamond$-PZT(58/42)Fe0.5; O-PZT(58/42)Fe1.0. quent poling step monotonously lowers permittivity and loss. More importantly, the data indicate that the loss maximum located around $f_{u}$ gets gradually suppressed remaining in its original position with respect to the frequency axis. Finally, it completely disappears from the spectrum in well poled samples. Meanwhile, the second peak gets revealed from the feature seen as a hump on the loss spectrum of unpoled sample. Close proximity between $f_{p}$ and the hump position indicates that domain configuration has no influence on corresponding polarization mechanism. The presence of such hump [seen also in PZT(58/42)Fe0.1] itself suggests that two different dissipation processes take place simultaneously in unpoled specimens. Therefore, we will discuss these processes separately.

Let us begin with poled samples. For specimens of given composition $f_{p}$ proved to be reproducible within the measurement resolution and independent of sample dimensions. As $f_{p}$ tends to be higher for the samples with finer microstructure, an obvious choice was to look for its correlation with average grain size $\bar{D}$. We find it more convenient, however, to plot the acoustic wavelength $\lambda_{A}$, which corresponds to $f_{p}$, as a function of $\bar{D}$, as it accounts for the variation of sound velocity $V_{s}$ among different compositions $\left(\lambda_{A}\right.$ $=V_{s}\left(f_{p}\right)$. The results are shown in Fig. 3. The solid line represents here the longitudinal resonance condition $\bar{D}=\lambda_{A} / 2{ }^{17}$ It demonstrates reasonable agreement with the data for $\bar{D}$ $<11 \mu \mathrm{m}$. It is possible to go beyond this limit using a fit

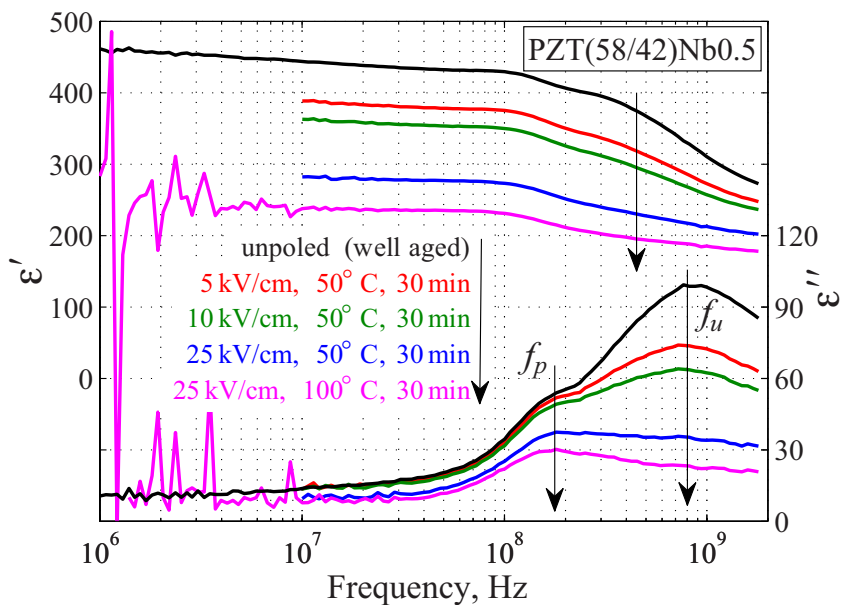

FIG. 2. (Color online) The influence of poling on PZT(58/42)Nb0.5 spectra. The arrows indicate evolution of spectra and corresponding consecutive poling conditions. 


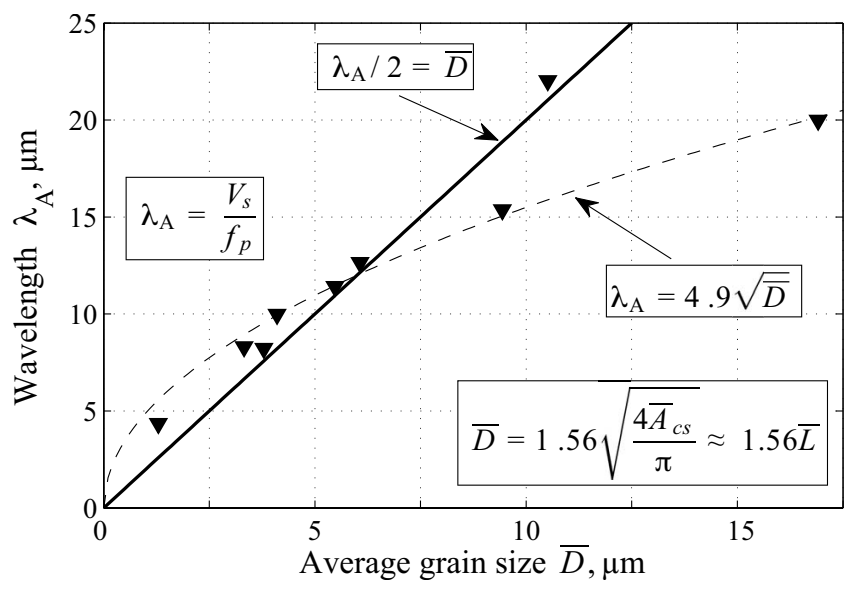

FIG. 3. The acoustic wavelength $\lambda_{A}$, which corresponds to the loss peak frequency $f_{p}$, vs average grain size $\bar{D}$. The solid line indicates the calculated longitudinal resonance condition: $\bar{D}=\lambda_{A} / 2$. The dashed line is the square root fit of the data.

based on square root function, which is shown in dashed line in Fig. 3. Though it does not provide any principal improvement over the linear dependence, as one of data points (at $10.5 \mu \mathrm{m})$ is still found considerably deviated from the fit. Square law could also indicate resonance of domains, whose width is reported to be proportional to $\sqrt{\bar{D}}$. ${ }^{9}$ We exclude this possibility because square fit obtained here would suggest domain width larger than $\bar{D}$. Alternatively, one could try to find the reason for observed deviation from the linear behavior for PZT(58/42) Nb0.2, which is the composition with the largest grain size. Indeed, there is a factor that, in our opinion, may explain it. Namely, it is known that the grains smaller than about $10 \mu \mathrm{m}$ tend to contain a single stack of lamellar domains, whereas larger grains split into several clusters. $^{9,18}$ At present it is not clear how well boundaries between such clusters can scatter acoustic waves and how they are affected by poling. However, it is not unreasonable to assume that a cluster of lamellas could behave as a grain with effective size that is smaller than the size of the whole grain. On condition that this assumption is correct, $f_{p}$ is expected to saturate for coarse-grain ceramics. Having that in mind, we can draw the following conclusion. The tendency for $\lambda_{A}$ to be linearly dependent on $\bar{D}$ considered together with the fact that $f_{p}$ does not depend on the poling state (i.e., domain pattern), indicates that the piezoelectric resonance of grains is responsible for the loss peak observed around $f_{p}$. This conclusion should be applied with the reservation that it concerns the ceramics with $\bar{D}_{e}$ smaller than about $11 \mu \mathrm{m}$.

Drastic suppression of dispersion around $f_{u}$ induced by poling supports the idea that it is of domain-related nature. The best explanation for such poling behavior can probably be offered by the model of the shear wave sound emission. ${ }^{6}$ It essentially implies constructive interference of acoustic waves in periodic domain structures. Poling is expected to break their periodicity, while domain wall density still remains unaffected. However, other domain wall processes cannot be excluded.

Much higher loss intensity found around $f_{u}$ compared to the intensity of loss peak around $f_{p}$ clearly demonstrates that the piezoelectric resonance of grains cannot be considered as the main mechanism responsible for strong microwave dispersion observed in ferroelectrics. On the other hand, due to the fact that loss associated with grain resonance dominates the loss in poled state, it can play the role of a frequency limiting factor for application sensitive to energy loss in piezoceramics.

The authors acknowledge financial support of the Swiss National Science Foundation Grant No. 200021-116038.

${ }^{1}$ G. Arlt, U. Böttger, and S. Witte, Ann. Phys. 506, 578 (1994).

${ }^{2}$ O. Kersten and G. Schmidt, Ferroelectrics 67, 191 (1986).

${ }^{3}$ M. P. McNeal, S.-J. Jang, and R. E. Newnham, J. Appl. Phys. 83, 3288 (1998).

${ }^{4}$ C. Kittel, Phys. Rev. 83, 458 (1951).

${ }^{5}$ N. A. Pertsev and G. Arlt, J. Appl. Phys. 74, 4105 (1993).

${ }^{6}$ G. Arlt, U. Böttger, and S. Witte, Appl. Phys. Lett. 63, 602 (1993).

${ }^{7}$ Y. Xi, H. McKinstry, and L. E. Cross, J. Am. Ceram. Soc. 66, 637 (1983).

${ }^{8}$ M. Maglione, R. Böhmer, A. Loidl, and U. T. Höchli, Phys. Rev. B 40, 11441 (1989).

${ }^{9}$ W. W. Cao and C. A. Randall, J. Phys. Chem. Solids 57, 1499 (1996).

${ }^{10}$ M. I. Morozov, Ph.D. thesis, EPFL, 2005.

${ }^{11}$ M. I. Morozov and D. Damjanovic, J. Appl. Phys. 104, 034107 (2008).

${ }^{12}$ ImageJ is available at http://rsbweb.nih.gov/ij/.

${ }^{13}$ M. I. Mendelson, J. Am. Ceram. Soc. 52, 443 (1969).

${ }^{14} \mathrm{~J}$. Grigas, Microwave Dielectric Spectroscopy of Ferroelectrics and Related Materials (Gordon and Breach, Amsterdam, 1996).

${ }^{15}$ R. G. Geyer, P. Kabos, and J. Baker-Jarvis, IEEE Trans. Instrum. Meas. 51, 383 (2002).

${ }^{16}$ IEEE Standard on Piezoelectricity, ANSI/IEEE Std 176-1987 edition, 1987.

${ }^{17}$ The other one-dimensional modes are expected to provide similar resonance criteria in given circumstances.

${ }^{18}$ G. Arlt, Ferroelectrics 104, 217 (1990). 